\title{
PERIODIC SOLUTIONS OF HYPERBOLIC PARTIAL DIFFERENTIAL EQUATIONS ${ }^{1}$
}

\author{
A. K. AZIZ
}

1. Introduction. We consider the normal form of the nonlinear partial differential equation of hyperbolic type in two independent variables:

$$
u_{x y}=f\left(x, y, u, u_{x}, u_{y}\right) .
$$

It is well known that, if in addition to usual smoothness conditions, the functions $f$ and the prescribed data $u_{0}(x)$ are periodic in $x$ with the same period $T$, then there exists a solution $u(x, y)$ of (1.1) satisfying $u(x, 0)=u_{0}(x), u(0, y)=v_{0}(y)+u_{0}(0)$, where $v_{0}(0)=0$ for $x \in(-\infty, \infty)$ and $y \in[-a, a]$. However, this solution is periodic only on the $x$-axis, but not necessarily in the strip $x \in(-\infty, \infty)$, $y \in[-a, a]$. Cesari in [1] has raised the question whether it is possible to choose $v_{0}(y)$ in such a way that $u(x, y)$ is periodic in $x$ for all $y \in[-a, a]$. Thus, we observe that the periodicity requirement $u(x+T, y)=u(x, y)$ leads to the integral equation:

$$
m(y)=\int_{0}^{T} f\left(x, y, u, u_{x}, u_{y}\right) d x=0
$$

in the unknown $v_{0}(y)$, where $u$ is thought to depend on $v_{0}(y)$ only. lem:

In this connection in [1] Cesari has considered the following prob-

$$
\begin{aligned}
& u_{x y}=f\left(x, y, u, u_{x}, u_{y}\right)-\frac{1}{T} \int_{0}^{T} f\left(x, y, u, u_{x}, u_{y}\right) d x \text {, } \\
& u(x+T, y)=u(x, y), \quad u(x, 0)=u_{0}(x), \\
& u(0, y)=u_{0}(0)+v_{0}(y), \quad v_{0}(0)=0,
\end{aligned}
$$

for $x \in(-\infty, \infty)$ and $y \in[-a, a]$, where $u_{0}, v_{0}$ are prescribed functions and $a$ is a real constant. The function $f$ in addition to being bounded, continuous in all arguments and periodic in $x$ with period $T$, satisfies a Lipschitz condition in the last two arguments, i.e.

$$
|f(x, y, u, p, q)-f(x, y, u, \bar{p}, \bar{q})| \leqq M|p-\bar{p}|+b|q-\bar{q}| .
$$

It is shown that if $u_{0}(x)$ and $v_{0}(y) \in C^{\prime}$ and if

Received by the editors May 3, 1965.

1 This research was supported in part by the NSF Grant GP 4493 and in part by the U.S. Naval Ordnance Laboratory, White Oak, Maryland. 


$$
2 b T<1, \quad M a<1,
$$

then there exists a solution of (1.2) satisfying (1.3). It is also proved in [1] that if $f$ satisfies a Lipschitz condition in the last 3 variables then the solution is unique and depends continuously on the initial data.

Cesari [3] has more recently considered the problem

$$
\begin{aligned}
u_{x y}= & f\left(x, y, u, u_{x}, u_{y}\right)-\frac{1}{T} \int_{0}^{T} f\left(x, y, u, u_{x}, u_{y}\right) d x \\
& -\frac{1}{T} \int_{0}^{T} f\left(x, y, u, u_{x}, u_{y}\right) d y \\
& +\frac{1}{T^{2}} \int_{0}^{T} \int_{0}^{T} f\left(x, y, u, u_{x}, u_{y}\right) d x d y, \\
u(x+T, y)= & u(x, y), \quad u(x, y+T)=u(x, y), \\
u(x, 0)= & u_{0}(x), \quad u(0, y)=u_{0}(0)+v_{0}(y), \quad v_{0}(0)=0 .
\end{aligned}
$$

Under assumptions similar to those stated above, existence, uniqueness, and continuous dependence on initial data for the solution of (1.6), (1.7) are shown. The problem of existence of a periodic solution of (1.1) in the strip $x \in(-\infty, \infty), y \in[-a, a]$, now leads to the question whether one can determine $v_{0}(y)$ in such a way that $m(y)=0$. A similar but more difficult question arises in the case where the periodic solutions of (1.1) are sought in the entire plane. In [2], and [3] these questions are discussed at length.

In the present paper we shall be concerned with the existence of a solution (not necessarily unique) to problem (1.2), (1.3) and (1.6), (1.7). The question of uniqueness, continuous dependence on initial data, will be dealt with in a separate note.

In $\$ 2$, we give two existence theorems, Theorems 2.1 and 2.2, where the condition (1.4) is replaced by a more general condition, i.e.

$$
|f(x, y, u, p, q)-f(x, y, u, \bar{p}, \bar{q})| \leqq \omega_{1}(|p-\bar{p}|)+\omega_{2}(|q-\bar{q}|)
$$

(for the smoothness assumptions concerning the functions $\omega_{i}(t)$, $i=1,2$, see the statements of Theorems $2.1,2.2$ below). We note that if $\omega_{i}(t), i=1,2$, are linear, then (1.8) reduces to (1.4). Thus (1.8) contains the Lipschitz condition as a special case (see Corollaries 2.1, 2.2 below). It also appears that the introduction of condition (1.8) simplifies the proofs and makes the results more explicit.

2. Existence of a solution. In this section we shall give sufficient conditions for the existence of a solution for the following problems: 


$$
\begin{array}{rlrl}
u_{x y}(x, y) & =f\left(x, y, u, u_{x}, u_{y}\right)-\frac{1}{T} \int_{0}^{T} f\left(x, y, u, u_{x}, u_{y}\right) d x \\
u(x+T, y) & =u(x, y), & u(x, 0) & =\phi_{1}(x), \\
u(0, y) & =\psi_{1}(y), & \phi_{1}(0) & =\psi_{1}(0),
\end{array}
$$

and

$$
\begin{aligned}
& u_{x y}(x, y)=f\left(x, y, u, u_{x}, u_{y}\right)-\frac{1}{T} \int_{0}^{T} f\left(x, y, u, u_{x}, u_{y}\right) d x \\
& -\frac{1}{T} \int_{0}^{T} f\left(x, y, u, u_{x}, u_{y}\right) d y \\
& +\frac{1}{T^{2}} \int_{0}^{T} \int_{0}^{T} f\left(x, y, u, u_{x}, u_{y}\right) d x d y, \\
& u(x+T, y+T)=u(x, y), \quad u(x, 0)=\phi_{2}(x), \\
& u(0, y)=\psi_{2}(y), \quad \phi_{2}(0)=\psi_{2}(0),
\end{aligned}
$$

We first consider the problems (2.2), (2.3) and (2.4), (2.5) for $x, y \in R_{1}$ $=[0, T] \times[-a, a]$ and $R_{2}=[0, T] \times[0, T]$, where $a$ and $T>0$ are given constants. Once the existence of solutions of the above problems are established in $R_{1}$ and $R_{2}$, then by the use of periodicity of $f$, we extend these solutions for $x \in(-\infty, \infty)$ and $y \in(-\infty, \infty)$.

We observe that if $u(x, y)$ and $v(x, y)$ are solutions of $(2.2),(2.3)$ and (2.4), (2.5) respectively and $s(x, y)=u_{x y}, r(x, y)=v_{x y}$, then $s \in C\left(R_{1}\right)$ and $r \in C\left(R_{2}\right)$, where $C\left(R_{1}\right)$ and $C\left(R_{2}\right)$ denote the space of continuous functions on $R_{1}$ and $R_{2}$ respectively. It is easily verified that the functions $s(x, y)$ and $r(x, y)$ satisfy the functional equations

$$
\begin{aligned}
& s(x, y)=F\left(x, y, B_{1} s, B_{2} s, B_{3} s\right), \\
& r(x, y)=G\left(x, y, B_{1} r, B_{2} r, B_{3} r\right),
\end{aligned}
$$

where for any $\theta \in C\left(R_{1}\right)$ or $\theta \in C\left(R_{2}\right)$

$$
\begin{aligned}
B_{1} \theta(x, y) & =\int_{0}^{x} \int_{0}^{y} \theta(\xi, \eta) d \xi d \eta+u(x, 0)+u(0, y)-u(0,0), \\
(2.8) \quad B_{2} \theta(x, y) & =\int_{0}^{y} \theta(x, \eta) d \eta+u_{x}(x, 0), \\
B_{3} \theta(x, y) & =\int_{0}^{x} \theta(\xi, y) d \xi+u_{y}(0, y),
\end{aligned}
$$

and 


$$
\begin{aligned}
F(x, y, u, p, q)= & f(x, y, u, p, q)-\frac{1}{T} \int_{0}^{T} f(x, y, u, p, q) d x \\
G(x, y, u, p, q)= & f(x, y, u, p, q)-\frac{1}{T} \int_{0}^{T} f(x, y, u, p, q) d x \\
& -\frac{1}{T} \int_{0}^{T} f(x, y, u, p, q) d y \\
& +\frac{1}{T^{2}} \int_{0}^{T} \int_{0}^{T} f(x, y, u, p, q) d x d y
\end{aligned}
$$

Conversely if $s \in C\left(R_{1}\right)$ and $r \in C\left(R_{2}\right)$ satisfy (2.6) and (2.7) and $u(x, 0), u(0, y) \in C^{\prime}$ are prescribed, then the functions

$$
u(x, y)=\left(B_{1} s\right)(x, y), \quad v(x, y)=\left(B_{1} r\right)(x, y)
$$

are solutions of $(2.2),(2.3)$ and (2.4), (2.5) respectively. Hence to show the existence of solutions of our problems it is sufficient to prove that the operators $J_{1}$ and $J_{2}$ defined by

$$
\begin{gathered}
\left(J_{1} s\right)(x, y)=F\left(x, y, B_{1} s, B_{2} s, B_{3} s\right), \\
\left(J_{2} r\right)(x, y)=G\left(x, y, B_{1} r, B_{2} r, B_{3} r\right),
\end{gathered}
$$

have fixed points in $C\left(R_{1}\right)$ and $C\left(R_{2}\right)$ respectively. To this end we assume that the following hypotheses hold

$\mathrm{H}_{1}: f(x, y, u, p, q)$ is continuous for $x \in(-\infty, \infty), y \in[-a, a]$ and $(u, p, q) \in(-\infty, \infty)$ and periodic in $x$ with period $T ; f$ is uniformly bounded $\left(|f| \leqq L_{1}\right)$ and satisfies the condition

$$
\begin{aligned}
& |f(x, y, u, p, q)-f(x, y, u, \bar{p}, \bar{q})| \\
& \quad \leqq \omega_{1}\left(|p-\bar{p}|+\omega_{2}(|q-\bar{q}|),\right.
\end{aligned}
$$

where $\omega_{i}(t)$ for $i=1,2$ are subadditive, nondecreasing, nonnegative, continuous for $t \in[0, \infty)$, and vanishing only for $t=0$.

$\mathrm{H}_{2}$ : The functions $\phi_{1}(x), \psi_{1}(y)$ are of class $C^{\prime}$ for $x \in(-\infty, \infty)$ and $y \in[-a, a]$ respectively, $\phi_{1}(x)$ is periodic with period $T$ and $\phi_{1}(0)$ $=\psi_{1}(0)$.

$\mathrm{H}_{1}^{\prime}: f(x, y, u, p, q)$ is continuous for $(x, y, u, p, q) \in(-\infty, \infty)$ and periodic in $x$ and in $y$ with period $T . f$ is uniformly bounded $\left(|f| \leqq L_{2}\right)$ and satisfies (2.13).

$\mathrm{H}_{2}^{\prime}: \phi_{2}(x), \psi_{2}(y)$ are periodic in $x$ and in $y$ respectively and of class $C^{\prime}$ for $x, y \in(-\infty, \infty)$, with $\phi_{2}(0)=\psi_{2}(0)$.

Let 


$$
\begin{aligned}
& N_{1}=\max _{x, y \in R_{1}}\left\{\left|\phi_{1}(x)\right|,\left|\psi_{1}(y)\right|,\left|\phi_{1}^{\prime}(x)\right|,\left|\psi_{1}^{\prime}(y)\right|\right\}, \\
& N_{2}=\max _{x, y \in R_{2}}\left\{\left|\phi_{2}(x)\right|,\left|\psi_{2}(y)\right|,\left|\phi_{2}^{\prime}(x)\right|,\left|\psi_{2}^{\prime}(y)\right|\right\}, \\
& K_{1}=\max \left\{a T M_{1}+3 N_{1}, T M_{1}+N_{1}, a M_{1}+N_{1}\right\}, \quad M_{1} \geqq 2 L_{1}, \\
& K_{2}=\max \left\{T^{2} M_{2}+3 N_{2}, T M_{2}+N_{2}, T M_{2}+N_{2}\right\}, \quad M_{2} \geqq 4 L_{2},
\end{aligned}
$$

where $M_{1}$ is defined by (2.19) below, and $M_{2}$ is similarly defined. From the continuity of the functions $f(x, y, u, p, q), \phi_{1}(x), \psi_{1}(y)$, $\phi^{\prime}(x)$ and $\psi^{\prime}(y)$ for $x, y \in R_{1}$ and $u, p, q \in\left[-K_{1}, K_{1}\right]$, it follows that they possess a common modulus of continuity i.e. there exists a function $\Omega(\delta)$ such that

$$
\begin{aligned}
& \left\{|f(x, y, u, p, q)-f(\bar{x}, \bar{y}, \bar{u}, \bar{p}, \bar{q})|,\left|\phi_{1}(x)-\phi_{1}(\bar{x})\right|\right. \\
& \left.\left|\phi_{1}^{\prime}(x)-\phi_{1}^{\prime}(\bar{x})\right|,\left|\psi_{1}(y)-\psi_{1}(\bar{y})\right|,\left|\psi_{1}^{\prime}(y)-\psi_{1}^{\prime}(\bar{y})\right|\right\} \leqq \Omega(\delta),
\end{aligned}
$$

whenever $|x-\bar{x}|+|y-\bar{y}|+|u-\bar{u}|+|p-\bar{p}|+|q-\bar{q}| \leqq \delta$ for $(x, y)$, $(\bar{x}, \bar{y}) \in R_{1}, u, p, q, \bar{u}, \bar{p}, \bar{q} \in\left[-K_{1}, K_{1}\right]$, and $\Omega(\delta)$ is nondecreasing, nonnegative, continuous and vanishing for $\delta=0$.

Let

$$
\begin{aligned}
& \Omega_{1}(\delta)=\Omega\left(\left[1+M_{1} a\right] \delta+\Omega(\delta)\right)+\omega_{1}(\Omega(\delta))+\omega_{2}\left(M_{1} \delta\right), \\
& \Omega_{2}(\delta)=2\left\{\Omega\left(\left[1+M_{1} T\right] \delta+\Omega(\delta)\right)+\omega_{1}\left(M_{1} \delta\right)+\omega_{2}(\Omega(\delta))\right\}, \\
& \pi_{1}(\delta)=2\left\{\pi\left(\left[1+M_{2} T\right] \delta+\pi(\delta)\right)+\omega_{1}(\pi(\delta))+\omega_{2}\left(M_{2} \delta\right)\right\}, \\
& \pi_{2}(\delta)=2\left\{\pi\left(\left[1+M_{2} T\right] \delta+\pi(\delta)\right)+\omega_{1}\left(M_{2} \delta\right)+\omega_{2}(\pi(\delta))\right\},
\end{aligned}
$$

where $\pi(\delta)$ is the common modulus of continuity of $f(x, y, u, p, q)$, $\phi_{2}(x), \phi_{2}^{\prime}(x), \psi_{2}(y), \psi_{2}^{\prime}(y)$ for $x, y \in R_{2}, u, p, q \in\left[-K_{2}, K_{2}\right]$.

Now we state our main results:

THEOREM 2.1. If

(1) the hypotheses $\mathrm{H}_{1}$ and $\mathrm{H}_{2}$ hold,

(2) the solutions $\rho_{1}(t, \delta)$ and $\rho_{2}(t, \delta)$ of the equations

$$
\begin{aligned}
\rho_{1}(t, \delta)= & \Omega_{1}(\delta)+\omega_{1}\left(\int_{0}^{t} \rho_{1}(\tau, \delta) d \tau\right), \quad t \geqq 0, \\
\rho_{2}(t, \delta)= & \Omega_{2}(\delta)+\omega_{2}\left(\int_{0}^{t} \rho_{2}(\tau, \delta) d \tau\right) \\
& +\frac{1}{T} \int_{0}^{T} \omega_{2}\left(\int_{0}^{t} \rho_{2}(\tau, \delta) d \tau\right) d t, \quad t \in[0, T]
\end{aligned}
$$

are such that 


$$
\lim _{\delta \rightarrow 0} \rho_{i}(t, \delta)=0, \quad i=1,2, \quad \text { uniformly in } t
$$

then

(3) there exists a solution $u(x, y)$ of (2.2), (2.3) in the strip $|x|<\infty$, $|y| \leqq a$.

THEOREM 2.2. If

(1) the hypotheses $\mathrm{H}_{1}^{\prime}$ and $\mathrm{H}_{2}^{\prime}$ hold

(2) the solutions $\bar{\rho}_{1}(t, \delta)$ and $\bar{\rho}_{2}(t, \delta)$ of the equations

$$
\begin{aligned}
\bar{\rho}_{1}(t, \delta)= & \pi_{1}(\delta)+\omega_{1}\left(\int_{0}^{t} \bar{\rho}_{1}(\tau, \delta) d \tau\right) \\
& +\frac{1}{T} \int_{0}^{T} \omega_{1}\left(\int_{0}^{t} \bar{\rho}_{1}(\tau, \delta) d \tau\right) d t
\end{aligned}
$$

$$
\begin{aligned}
\bar{\rho}_{2}(t, \delta)= & \pi_{2}(\delta)+\omega_{2}\left(\int_{0}^{t} \bar{\rho}_{2}(\tau, \delta) d \tau\right) \\
& +\frac{1}{T} \int_{0}^{T} \omega_{2}\left(\int_{0}^{t} \bar{\rho}_{2}(\tau, \delta) d \tau\right) d t, \quad t \in[0, T],
\end{aligned}
$$

are such that

$$
\lim _{\delta \rightarrow 0} \bar{\rho}_{i}(t, \delta)=0, \quad i=1,2, \quad \text { uniformly in } t
$$

then

(3) there exists a solution $u(x, y)$ of (2.4), (2.5) in the entire plane $|x|<\infty,|y|<\infty$.

Proof of Theorem 2.1. To prove our theorem we show that the operator $J_{1}$ defined by $(2.11)$ has a fixed point in $C\left(R_{1}\right)$. We note since $f$ is bounded there exists a constant $M_{1}$ such that $\|s\| \leqq M_{1}$ implies $\left\|J_{1} s\right\| \leqq M_{1}$ for $s \in C\left(R_{1}\right)$.

Now let

$$
\begin{array}{r}
S=\left\{s \in C\left(R_{1}\right):\|s\| \leqq M_{1},|s(x, y)-s(\bar{x}, \bar{y})| \leqq \rho_{1}(y,|x-\bar{x}|)\right. \\
+\rho_{2}(\bar{x},|y-\bar{y}|),|x-\bar{x}|+|y-\bar{y}| \leqq \delta, \\
\left.(x, y),(\bar{x}, \bar{y}) \in R_{1}, \delta>0, M_{1} \geqq 2 L_{1}\right\},
\end{array}
$$

where the functions $\rho_{i}$ are solutions of (2.17). It follows easily from the definition of $S$, the properties of $\rho_{i}$ and Arzela's theorem that $S$ is closed, convex and compact. Next we show that $J_{1} S \subset S$. The boundedness of $f$ implies $\left\|J_{1} s\right\| \leqq M_{1}$. Moreover from the definition of $J_{1}$ it follows that 


$$
\begin{aligned}
&\left|\left(J_{1}\right)(x, y)-\left(J_{1} s\right)(\bar{x}, y)\right| \\
& \leqq \Omega\left(|x-\bar{x}|+\left|\left(B_{1} s\right)(x, y)-\left(B_{1} s\right)(\bar{x}, y)\right|\right) \\
& \quad+\omega_{1}\left(\left|\left(B_{2} s\right)(x, y)-\left(B_{2} s\right)(\bar{x}, y)\right|\right) \\
&+\omega_{2}\left(\left|\left(B_{3} s\right)(x, y)-\left(B_{3} s\right)(\bar{x}, y)\right|\right) .
\end{aligned}
$$

We note that

$$
\begin{aligned}
& \left|B_{1} s(x, y)-B_{1} s(\bar{x}, y)\right| \leqq M_{1} a|x-\bar{x}|+\Omega(|x-\bar{x}|), \\
& \left|B_{2} s(x, y)-B_{2} s(\bar{x}, y)\right| \leqq \int_{0}^{y}|s(x, \eta)-s(\bar{x}, \eta)| d \eta+\Omega(|x-\bar{x}|), \\
& \left|B_{3} s(x, y)-B_{3} s(\bar{x}, y)\right| \leqq M_{1}|x-\bar{x}| .
\end{aligned}
$$

Therefore

$$
\begin{aligned}
& \left|J_{1} s(x, y)-J_{1} s(\bar{x}, y)\right| \\
& \quad \leqq \Omega_{1}(|x-\bar{x}|)+\omega_{1}\left(\int_{0}^{y} \rho_{1}(\eta,|x-\bar{x}|) d \eta\right) \\
& \quad=\rho_{1}(y,|x-\bar{x}|) .
\end{aligned}
$$

Similarly we have

$$
\begin{aligned}
& \left|J_{1} s(\bar{x}, y)-J_{1} s(\bar{x}, \bar{y})\right| \\
& \leqq \Omega_{2}(|y-\bar{y}|)+\omega_{2}\left(\int_{0}^{\bar{x}} \rho_{2}(\xi,|y-\bar{y}|) d \xi\right) \\
& \quad+\frac{1}{T} \int_{0}^{T} \omega_{2}\left(\int_{0}^{\bar{x}} \rho_{2}(\xi,|y-\bar{y}|) d \xi\right) d \bar{x}=\rho_{2}(\bar{x},|y-\bar{y}|) .
\end{aligned}
$$

From (2.21) and (2.22) it follows that

$$
\left|J_{1} s(x, y)-J_{1} s(\bar{x}, \bar{y})\right| \leqq \rho_{1}(y,|x-\bar{x}|)+\rho_{2}(\bar{x},|y-\bar{y}|) .
$$

Hence $J_{1} S \subset S$.

It is easily seen that $J_{1}$ is continuous on $S$. Therefore by Schauder's Theorem [5], there exists an $s_{0} \in S$ such that $J_{1} s_{0}=s_{0}$, which in turn implies the existence of a solution $u(x, y)$ of (2.2), (2.3) in $R_{1}$. By extending both $u(x, y)$ and $f(x, y, u, p, q)$ for $|x|<\infty,|y| \leqq a$, $|u|,|p|,|q| \leqq K_{1}$, by means of periodicity of $f$ of period $T$ in $x(2.2)$, (2.3) is satisfied in the strip $|x|<\infty,|y| \leqq a$.

Proof of Theorem (2.2) is precisely the same as the proof of Theorem 2.1 except that the operator $J_{1}$ is replaced by the operator $J_{2}$ defined by (2.12) and in the definition (2.19) of the set $S, \rho_{i}$ are replaced by $\bar{\rho}_{i}$. 
Now we state two corollaries of the above theorems which contain Theorem I of [1] and Theorem I of [3].

Corollary 2.1. If

(1) hypotheses (1) of Theorem 2.1 hold,

(2) $\omega_{1}(t)=M t, \omega_{2}(t)=b t, M, b>0$,

(3) $e^{b T}<2 b T+1$, then

(4) there exists a solution $u(x, y)$ of (2.2), (2.3) in the strip $|x|<\infty$, $|y| \leqq a$.

Corollary 2.2. If

(1) hypotheses (1) of Theorem 2.2 hold,

(2) $\omega_{1}(t)=b_{1} t, \omega_{2}(t)=b_{2} t, b_{1}, b_{2}>0$,

(3) $e^{b_{1} T}<2 b_{1} T+1, e^{b_{2} T}<2 b_{2} T+1$, then

(4) there exists a solution $u(x, y)$ of (2.4), (2.5) in the entire plane $|x|<\infty,|y|<\infty$.

We give only the proof of Corollary 2.1. The proof of Corollary 2.2 is similar.

Proof of Corollary 2.1. To prove this corollary we need only show that (2) and (3) imply (2.17) of Theorem 2.1. It follows immediately from hypotheses (2) that (2.17) is equivalent to the following differential equations:

$$
\begin{aligned}
& r_{1}^{\prime}(t, \delta)=\Omega_{1}(\delta)+M r_{1}(t, \delta), \quad r_{1}(0, \delta)=0 \\
& r_{2}^{\prime}(t, \delta)=\Omega_{2}(\delta)+b r_{2}(t, \delta)+\frac{b}{T} \int_{0}^{T} r_{2}(\tau, \delta) d \tau, \quad r_{2}(0, \delta)=0
\end{aligned}
$$

where

$$
r_{1}(t, \delta)=\int_{0}^{t} \rho_{1}(\tau, \delta) d \tau, \quad r_{2}(t, \delta)=\int_{0}^{t} \rho_{2}(\tau, \delta) d \tau .
$$

We have at once

$$
r_{1}(t, \delta)=\frac{\Omega_{1}(\delta)}{M}\left(e^{M t}-1\right), \quad t \geqq 0 .
$$

The continuity of $\Omega_{1}(\delta)$ together with $\Omega_{1}(0)=0$ imply that $\lim _{\delta \rightarrow 0} r_{1}(t, \delta)=0$, and nonnegativity of $\rho_{1}(t, \delta)$ implies that $\lim _{\delta \rightarrow 0} \rho_{1}(t, \delta)=0$. Similarly (2.24) has the solution

$$
r_{2}(t, \delta)=\left(\frac{\Omega_{2}(\delta)}{b}+\frac{1}{T} \int_{0}^{T} r_{2}\right),(t, \delta) d t\left(e^{b t}-1\right), \quad t \in[0, T]
$$


provided

$$
\int_{0}^{T} r_{2}(t, \delta) d t\left[\frac{2 b T+1-e^{b T}}{b T}\right]=\frac{\Omega_{2}(\delta)}{b^{2}}\left[e^{b T}-b T-1\right] .
$$

Since (2.26) must hold for all $\delta \geqq 0$, and since $r_{2}(t, \delta)$ is nonnegative, (2.26) is satisfied if

$$
e^{b T}<2 b T+1 .
$$

Now from (2.25) and (2.26) we have

$$
r_{2}(t, \delta)=\frac{\Omega_{2}(\delta)}{b} C\left(e^{b t}-1\right),
$$

where

$$
C=\frac{e^{b T}-b T-1}{-e^{b T}+2 b T+1}+1 .
$$

Equation (2.28), together with continuity of $\Omega_{2}(\delta)$ for $\delta \geqq 0, \Omega_{2}(0)=0$ and nonnegativity of $\rho_{2}(t, \delta)$ imply $\lim _{\delta \rightarrow 0} \rho_{2}(t, \delta)=0$. Thus if $(2.27)$ holds all the hypotheses of Theorem 2.1 are satisfied and the assertion of Corollary 2.1 follows from Theorem 2.1.

3. Concluding remarks. We note that the hypotheses (3) of Corollaries 2.1 and 2.2 are satisfied for $2 b T<2.5,2 b_{i} T<2.5, i=1,2$. The restriction $M a<1$ in Theorem I of [1] is not required in Corollary 2.1 and the restrictions $2 b T<1$ can be replaced by $2 b T<2.50$. Similarly the restrictions $2 b_{i} T<1, i=1,2$ of Theorem 1 of [3] can be replaced by $2 b_{i} T<2.50, i=1,2$.

We also observe that if the function $f$ in Corollaries 2.1, 2.2 satisfies a Lipschitz condition in the last three arguments $u, p, q$, i.e. $|f(x, y, u, p, q)-f(x, y, \bar{u}, \bar{p}, \bar{q})| \leqq b_{0}|u-\bar{u}|+b_{1}|p-p|+b_{2}|q-\bar{q}|$, then the existence of the unique solutions of problems (2.2), (2.3) and (2.4), (2.5) follow immediately from Banach's contraction principle applied to the operators $J_{1}$ and $J_{2}$, provided we impose the restrictions:

$$
2\left(b_{0} a T+b_{1} a+b_{2} T\right)<1, \quad \text { in Corollary } 2.1,
$$

and

$$
4 T\left(b_{0} T+b_{1}+b_{0}\right)<1, \quad \text { in Corollary } 2.2 .
$$

\section{BIBLIOGRAPHY}

1. L. Cesari, Periodic solutions of hyperbolic partial differential equations, Inter- 
nat. Sympos. on Nonlinear Differential Equations and Nonlinear Mechanics, Colorado Springs, 1961, pp. 33-57, Academic Press, New York, 1963.

2. - A criterion for the existence in a strip of periodic solutions of hyperbolic partial differential equations, Rep. No. 11, 1964, Department of Mathematics, Univ. of Michigan, Ann Arbor, Mich.

3. - Existence in the large of periodic solutions of hyperbolic partial differential equations, Rep. No. 12, 1964, Department of Mathematics, Univ. of Michigan, Ann Arbor, Mich.

4. - The implict function theorem in functional analysis, Rep. No. 10, 1964, Department of Mathematics, Univ. of Michigan, Ann Arbor, Mich.

5. J. P. Schauder, Der Fixpunktsatz in Funktionalraumen, Studia Math. 2 (1930), 171-180.

Georgetown University 